\title{
Anabases
}

ANABASES Traditions et réceptions de l'Antiquité

$20 \mid 2014$

Varia

\section{En guise de catalexe}

\section{Philippe Brunet}

\section{OpenEdition}

Journals

Édition électronique

URL : http://journals.openedition.org/anabases/5093

DOI : 10.4000/anabases.5093

ISSN : 2256-9421

\section{Éditeur}

E.R.A.S.M.E.

\section{Édition imprimée}

Date de publication : 1 novembre 2014

Pagination : 279-285

ISSN : 1774-4296

\section{Référence électronique}

Philippe Brunet, «En guise de catalexe », Anabases [En ligne], 20 | 2014, mis en ligne le 01 novembre

2017, consulté le 21 octobre 2019. URL : http://journals.openedition.org/anabases/5093 ; DOI

10.4000/anabases.5093 


\section{Principes de scansion de l'hexamètre en français}

Dans les ReCréations françAises de l'hexamètre qui ont vu le jour ces dernières années ${ }^{1}$, la scansion est éprouvée à travers une expérience qui associe quatre principes : imitation verticale de la forme grecque ou latine, imitation horizontale des autres pratiques advenues dans les littératures modernes, élaboration de la forme à l'intérieur de la langue (non sans confrontation avec la prosodie traditionnelle), et expérimentation orale.

L'imitation verticale est le principe qui fonde la forme nouvelle dans sa mîmêsis, c'est-à-dire dans l'activation d'un principe rythmique et poétique qui ne valait jusquelà qu'à l'intérieur de la poésie grecque : ainsi procédèrent les premiers poètes latins instaurant la forme grecque dans leur littérature encore non mesurée jusque-là. Cette verticalité est ressentie dans l'idéalisation du modèle grec : la distance fait le désir d'hexamètre et le succès de la nouvelle scansion, réalisée dans les pas de la première.

L'imitation horizontale procède d'une comparaison des prosodies, des métriques, des expériences de scansion. Il est toujours intéressant d'avoir conscience de ce qui se fait ailleurs dans les autres langues, proches ou non. C'est un des intérêts de la rencontre "Homère en hexamètres " que de nous rendre tous contemporains les uns des autres, indépendamment de la chronologie. Homère devient lui-même à ce titre le premier de nos contemporains. Il est lui-même " au travail », comme Jean Irigoin se plaisait à

1 La traduction pionnière d'A. Markowicz, Le Livre de Catulle, Lausanne 1985 ; nos traductions : Poèmes et fragments de Sappho, Paris 1991, Batrachomyomachie, Paris 1998, Hésiode, Euvres, Paris 1999, l'Iliade orale des années 2005-2006, l'Iliade publiée au Seuil en 2010 et 2012 ; les mises en scènes de l'Odyssée et du répertoire dramatique avec le groupe Démodocos depuis 1995 ; on peut entendre ici et là en lecture les traductions en cours d'A. Münch (Virgile, Géorgiques) et de G. Boussard (Lucrèce, La Nature des choses). 
camper les poètes anciens ${ }^{2}$. Il ménage sa langue, façonne son matériau, triche, joue, régule, adapte les mots, utilise le rythme et le retour périodique de la marque rythmique pour façonner son épopée.

CEuvrant à son tour dans sa langue avec un principe comparable, sinon analogue, le poète-traducteur se trouve dans la même situation qu'Homère : façonner du matériau prosodique, rythmer de l'expression récurrente, tisser du son autour du nom qu'il accueille en le transcrivant. L'élaboration de la forme à l'intérieur de la langue, en liaison et confrontation avec les formes prosodiques traditionnelles, syllabiques ou accentuelles, suscite deux types de commentaires : les poètes-traducteurs peuvent justifier leur pratique, faire visiter leur laboratoire, écritoire ou gueuloir ; les lecteurscritiques apportent une appréciation plus extérieure, peut-être plus objective, des prétentions affichées par les traducteurs, au vu de leurs " résultats".

Enfin, l'expérimentation orale est un élément essentiel de cette pratique. Elle n'est pas un élément de validation tenté après coup, mais le lieu même où se réalise la scansion, où s'élabore ce que l'analyse a pour but de décrire. Il ne faudrait pas non plus la réduire à un lieu d'auto-expérimentation : c'est le lieu de l'échange avec le public. Pas plus qu'on ne peut enregistrer l'épopée seul dans son studio, on ne peut dire les hexamètres sans recevoir l'écoute complice de l'autre. Pour incarner cet Autre, condition nécessaire à l'interprétation vocale, il suffit d'une personne privilégiée. L'écoute valide le retour des temps et des mots, et instaure le cercle comme image de l'epos. La solidarité de l'aède et de celui qui reçoit la parole façonne un lien, une culture commune, une sorte de paideia.

Menant parallèlement des recherches sur la métrique grecque ainsi que des essais de traduction française métrique du vers grec, j’ai tenté de résumer les règles de scansion de l'hexamètre homérique grec en vue d'une scansion automatisée ${ }^{3}$. Ces règles, une fois formulées, ont donné lieu à l'élaboration de programmes de scansion du vers grec $^{4}$, dont un des intérêts à mes yeux était le constat, souvent fait par les phonéticiens et métriciens, d'une grande richesse et irrégularité prosodiques. Peut-on former un vers sur l'instabilité rythmique que l'analyse prosodique, aussi bien que la pratique vocale, met en évidence ? L'autre avantage de ces scansions est de permettre

2 J. IrIgOIn, Le poète grec au travail, Paris 2009. J. IRIGOIN (1929-2006) est disciple d'A. DAIn, auteur du Traité de métrique grecque, Paris 1965.

3 Nos divers séminaires libres ou cours donnés à l'EPHE ou l'université de Rouen entre 1990 et 2010 .

4 Programme de scansion Scande élaboré par P. DAVID, utilisé pour notre thèse en 1992, et le programme Scandeð Chante écrit par G. de Rosny, 2010, et disponible sur l'Atelier de métrique grecque et latine, www.homeros.fr ; un autre programme, ScandełMarque, en cours d'élaboration, permettra de scander l'hexamètre français. Voir aussi, sur le même site, et réalisé par G. de Rosny également, le programme Longīs de macronisation du latin. 
la constitution de sous-ensembles triés, isosyllabiques, isométriques ou analogues dans le melos accentuel.

L'expérimentation orale, commencée sous la conduite de Stephen Daitz ${ }^{5}$ dans les années 1990, s'est prolongée avec la naissance d'une troupe de théâtre, Démodocos, où furent associées scansion de la langue originale et scansions en langue moderne, dans une alternance poétique, musicale ou théâtrale. À partir de 2009, date d'un séjour en Éthiopie où j'ai découvert l'existence de lyres, instruments analogues aux cordophones antiques, ma diction du grec s'est développée au rythme du plectre, selon un accordage prédéfini. En même temps que je développais la danse avec son jeu de pas ${ }^{6}$, je découvrais que le jeu du plectre avait la même fonction corporelle d'harmonisation rythmique et mélodique de la parole. Ici la traduction ne couvre qu'une partie du travail, qui traite à égalité les langues originales et les langues de traduction. Parole, drame, chant, danse, quelle que soit la langue, se donnent dans un rythme où le mot est indissociable du geste, le rythme verbal, inséparable du pied.

Le bureau du traducteur s'y trouve concurrencé par la scène où l'aède fait advenir la réalité métrique de son vers. Au fil des récitals et des ateliers, on put tester la diction, et expérimenter les différentes manières de marquer les temps de l'hexamètre, dans un parler tour à tour naturaliste, régulé, ou récité, dans une énonciation aédique ou chorale. La régulation nécessaire pour réaliser la cohérence rythmique du vers peut porter sur le lissage des accents, la périodicité des temps, l'égalisation plus ou moins grande des quantités. Selon les besoins de l'interprétation (discours, narration, chœur, la forme, aédique ou théâtralisée, impliquant un rapport au public différent), cette variabilité rythmique devient l'un des principaux outils de l'aède.

La dimension gestuelle et corporelle se trouve au cœur du travail de diction et de traduction. Je ne peux ici développer cet aspect mais il est fondamental. La battue selon le rythme engage le corps ; c'est une respiration qui s'instaure, qui se traduit en geste. Pour apprendre à dire, il faut marcher. Le musicien le sait bien, et l'aède, au départ, est un musicien. Le frappé du plectre sur les cordes de l'instrument sculpte le cadre du melos langagier. Cette battue organise un rapport entre le haut et le bas, entre l'arrière et l'avant, des repères essentiels pour comprendre l'hexamètre. À ce titre, l'hexamètre n'est qu'un exemple de mètre parmi d'autres, et comme tel, il ne saurait être appréhendé hors de son interprétation performative : son articulation relève d'une prise en compte globale, incluant une relation rythmique au monde, au temps, à l'Autre.

C'est au titre de ces différentes expériences que je peux proposer aujourd'hui de définir, par un déplacement d'une langue dans une autre, quelques principes de scansion de l'hexamètre. J'espère ainsi répondre à Gilles de Rosny, qui pense possible

5 S.G. DaITZ, The Iliad of Homer in Ancient Greek. Madison, CT : Audioforum, 1992. Autres références sur le site http://rhapsodes.fll.vt.edu/.

6 Voir, par exemple, Ph. BRUnet, « La voix et le geste au pouls de la Muse ", Approches 150, juin 2012, p. 141-54. 
de soumettre aussi l'hexamètre français à un programme de scansion automatisée, et en faire ainsi un objet d'étude et non plus le simple privilège d'un traducteur solitaire, certes partagé par quelques récitants et lecteurs complices.

C'est aussi l'accomplissement d'un rêve contenu dans le Livre de Catulle d'André Markowicz : faire exister la parole antique, lui donner un statut, une forme dans la littérature française. Dire et mémoriser Virgile en français sera bientôt possible autrement que dans les (beaux) décasyllabes de Joachim du Bellay; jouer les Tragiques pourra se faire dans une idée organique du drame, orchestique, moins inculte en tout cas que celles auxquelles nous étions condamnés par une approche auto-proclamée dans l'absolu « contemporaine ».

\section{Indifférence de la prosodie française ?}

C'est le reproche qu'on fait souvent à la langue, de ne pas avoir l'opposition de quantité qui existerait en grec ancien à l'état naturel, et de ne pas avoir non plus la ressource accentuelle que possèdent d'autres langues comme l'allemand ou le russe. À ces détracteurs, je me souviens qu'André Markowicz répondait en citant, au début des années 80 , les vers bien connus :

Maître Corbeau sur un arbre perché

Tenait en son bec un fromage...

Ces deux vers, un décasyllabe et un octosyllabe, mis bout à bout, font résonner une cadence dactylique, à sept temps marqués. Une attaque sur l'initiale amorce un rythme descendant, régulier, avec deux syllabes entre deux marques. Après la césure (la rime, en fait), on a une syllabe avant la nouvelle marque. Autrement dit, après la marque à la césure, le rythme se prolonge sur un rythme ascendant. Ce rythme n'est pas le fruit du hasard, puisqu'il est répété juste après, dans un parallélisme comique :

Maître Renard, par l'odeur alléché,

Lui tint à peu près ce langage...

C'est, à une marque près, le rythme de l'hexamètre dactylique. Cette scansion, qui nourrit de manière dynamique le syllabisme apparent de La Fontaine, a été utilisée en français à la Renaissance par des gens comme Mousset, l'auteur d'une Iliade perdue, Baïf, Jodelle, d'Aubigné, Buttet, avec une grande sensibilité à la langue et à la structure prosodique de ses syllabes. Fantôme récurrent dans les lettres françaises, trace prétendue invisible dans le vers syllabique, ce principe de marquage tonique a été développé, en 1985, par André Markowicz dans sa transposition des mètres de Catulle ${ }^{7}$. Je l'ai utilisé pour façonner le vers de l'épopée grecque archaïque. Cet hexamètre repose 
à la fois sur des qualités naturelles propres à la langue, sur le moule métrique dans lequel on contraint la langue, enfin sur la maîtrise aédique d'un principe de régulation rythmique.

Dans cet extrait de l'Iliade, que je reproduis dans le livre imprimé, plutôt qu'en produire ici de nouvelles variantes, je souligne les marques aux six temps forts de l'hexamètre.

Chante, Déesse, l'ire d'Achille Péléiade, ire funeste, qui fit la douleur de la foule achéenne, précipita chez Hadès, par milliers, les âmes farouches des guerriers, et livra leur corps aux chiens en pâture, 5 aux oiseaux en festin - achevant l'idée du Cronide -, depuis le jour où la discorde affronta l'un à l'autre Agamemnon, le souverain maître, et le divin Achille ! Qui, des dieux, déclencha l'affrontement des deux hommes? L'enfant de Zeus et Létô. Il jeta, courroucé par le maître,

10 sur l'armée, un mal odieux, dont les hommes moururent, puisque Chrysès, son prêtre, avait reçu un outrage d'Agamemnon. Le prêtre, venu jusqu'aux nefs achéennes pour délivrer sa fille, portait la rançon innombrable, arborait les emblèmes du dieu-des-cibles-lointaines,

15 haut sur le sceptre d'or, suppliait les Argiens de la foule, et surtout les deux Atréides, maitres des hommes :

"Vous, Atréides, et vous, Achéens aux jambières solides, puissent les dieux vous donner, les dieux vivant sur l'Olympe, d'anéantir la cité de Priam, de rentrer sur vos terres!

20 Mais libérez ma fille et prenez la rançon en échange : craignez le fils de Zeus, Apollon, dieu-des-cibles-lointaines! » Les Achéens, unanimes alors, d'une voix consentirent à respecter le prêtre, à garder la rançon éclatante, ce qui déplut au cour d'Agamemnon l'Atréide.

25 Il le chassa rudement, l'accabla de paroles terribles: "Si jamais je te vois, vieillard, près des creuses carènes ou t'attarder ce jour, ou plus tard revenir à la charge, sceptre et emblèmes du dieu te seront, à toi, inutiles. Je ne la rendrai pas : elle attendra la vieillesse

30 dans mon palais d'Argolide, loin du pays de ses pères, à manœuvrer la navette, à se pavaner sur ma couche ! Va! Ne m'irrite plus, si tu veux repartir sans dommage!" Le vieillard prit peur, obéit à son ordre. En silence, 
il longea le rivage de l'onde retentissante ${ }^{8} \ldots$

J'expliquerai maintenant la nature de ces différentes marques, placées sur les temps forts qui font la structure de l'hexamètre.

\section{Le schéma métrique et la marque}

Le vers de l'épopée se définit par le retour de six marques : la première marque, au temps fort, correspond à la syllabe initiale du vers. Puis deux syllabes sont non-marquées, au temps faible, avant la $2^{\mathrm{e}}$ syllabe marquée. Au lieu de deux syllabes non-marquées, on peut se contenter d'en avoir une seule. Cette équivalence d'une syllabe « longue » valant deux syllabes «brèves » au temps faible est caractéristique de ce vers. On a toujours une seule syllabe au temps dit fort.

On compte ainsi 5 mesures tantôt trisyllabes $(\mathrm{N}$ a b), tantôt dissyllabes ( $\mathrm{N} \mathrm{c}$, la $5^{\mathrm{e}}$ mesure étant plus rarement dissyllabe) et une $6^{\mathrm{e}}$ mesure strictement dissyllabe. Si les chiffres indiquent le numéro $\mathrm{N}$ de la syllabe au temps fort, et, au temps faible, les lettres $\mathrm{a}, \mathrm{b}$, les voyelles «brèves ", ou c, la voyelle « longue " unique :

$$
1 \text { a b } 2 \text { a b } 3 \text { ab } 4 \text { ab } 5 \text { a b } 6 \text { c }
$$

En indiquant les coupes les plus fréquentes :

$$
1 \mathrm{ab} 2 / \mathrm{ab} \mathrm{3/a/b} 4 / \mathrm{ab} / 5 \mathrm{ab} 6 \mathrm{c}
$$

Dans les langues anciennes, le groupe au temps faible, qu'il soit dissyllabe (a b) ou monosyllabe (c), est considéré comme équivalent. Le temps fort est en principe équivalent par sa durée au temps faible dans le vers grec, même si dans la réalité, il en va autrement. En fait, l'égalité de quantité est une illusion obtenue par l'alternance temps fort/temps faible. L'égalité entre les syllabes au temps fort est aussi une illusion obtenue par le retour de la pulsation rythmique. Le vers n'existe qu'en instituant un principe de régulation des quantités entre les différentes syllabes. Cette régulation maintient le système sans le rendre strictement homogène, et introduit des règles d'usage, des modes de diction : des principes et des exceptions.

Ainsi, l'hexamètre, de même que chacun de ses six pieds, commence toujours sur une syllabe longue. Cette syllabe longue est idéalement faite d'un élément vocalique long, mais peut être formée aussi d'un élément vocalique bref en syllabe fermée. On trouve également, à titre exceptionnel, une syllabe brève qu'on se gardera de vouloir à tout prix prolonger, comme dans ce célèbre début d'hexamètre, où la délicate Aphrodite s'adresse à son frère Arès ${ }^{9}$ :

8 Ph. Brunet (trad.), Homère, L'Iliade, Seuil, Paris 2010, Point Seuil, Paris 2012 (chant 1, $1-35)$.

9 Il. 5.359, cf. 4.155, 21.308. Voir, pour d'autres irrégularités prosodiques, nos articles sur homeros.fr. 
$\phi i ́ \lambda \epsilon \kappa a \sigma i \gamma \nu \eta \tau \epsilon \ldots$.

phile kasignête...

Le schéma normal $1 \mathrm{a} b 2 \mathrm{c} 3 \ldots$, c'est-à-dire $\mathrm{L}$ a $\mathrm{b} \mathrm{L} \mathrm{c} \mathrm{L}$, où $\mathrm{L}$ est une syllabe longue au temps fort, se trouve remplacé par B a b L c B..., si B signifie une syllabe brève. Les deux longues qui délimitent ce côlon de six syllabes très familier sont remplacées par des brèves marquées : brève exceptionnelle à l'initiale du vers, brève exceptionnelle à la césure penthémimère. Pour des raisons expressives, l'hexamètre contredit ce qu'il fait habituellement. La seule syllabe réellement longue au temps fort, -sig-, soulignée par l'intonation mélodique, n'est pas longue par nature. Et pour narguer toutes les lois de la prosodie, la seule voyelle authentiquement longue se trouve au temps faible. Ce début de vers, impossible, incohérent, n'est rendu acceptable que par le traitement expressif de l'exception ; le rythme ne saurait y contraindre les quantités en les allongeant, car, sinon, le vers deviendrait banal et maladroit. L'alternance thesis / arsis se maintient, que bien, que mal, et tout l'art vient de la difficulté. J'insiste sur ce contre-exemple pour montrer que l'hexamètre est loin de correspondre au schéma des manuels censé le représenter.

Mais en règle générale, pour commencer l'hexamètre, on peut utiliser une syllabe que la nature prédispose à porter l'accent ; ou une syllabe à laquelle on va donner un statut particulier. Il va donc falloir distinguer entre deux types de syllabes.

En français, le temps dit fort $(\mathrm{TF})$ subsume plusieurs phénomènes phonologiques tels que l'accent tonique, la quantité, l'intensité, l'intonation, ou la qualité particulière de la syllabe selon qu'elle est placée en fin de groupe ou à l'initiale du mot ou du groupe. La quantité est liée à la structure complexe de la syllabe (diphtongue, syllabe fermée voyelle + consonne VC\#, groupe voyelle + sonante VR\#, etc.). Comme en grec, cette quantité, extrêmement flexible, et insuffisante par nature dès que l'on cherche à obtenir des syllabes longues au moyen de voyelles brèves, demande qu'on institue une opposition entre deux types de syllabes. À ces divers titres, ces syllabes doivent ou peuvent acquérir une valeur prosodique marquée qu'on opposera, dans une opposition rythmique, à des éléments subsidiaires divers non valorisés au temps faible (Tf).

La valorisation de cet élément au TF implique un renforcement de la durée, de l'intensité, ou de l'intonation, dans des proportions très variables, qui relèvent autant de la langue que de l'interprétation vocale. Et cela, sans préjuger des horizons de la parole scandée, proférée, récitée, chantée. On ne dira pas que l'hexamètre doit être accentuel, ou quantitatif, dans une opposition entre un principe accentuel intensif, à l'œuvre dans les prosodies modernes, et un principe quantitatif idéalisé, celui du vers antique ou de certaines langues exotiques. Il va de soi que la quantité prônée chez les Anciens est travaillée par la régulation dans l'alternance périodique où consiste le rythmos.

La constitution de langues poétiques confiées à la voix, avec cette part d'hypnose suscitée par la réitération de schémas rythmiques et par leurs variations subtiles, risque d'éloigner cette parole de sa dimension naturelle, " oralité " très souvent vantée dans la littérature contemporaine, et de lui conférer une autre dimension vocale, incantatoire. 
En tout cas, c'est dans un conflit non tranché entre oralité brute et récitatif soutenu, régulé, et dans une suite d'échanges continus avec ce qu'entend le public devant qui les hexamètres sont dits, que se situe la tentative.

Quant aux éléments subsidiaires non valorisés (Tf), ils ne sont pas tous exactement unis dans la même faiblesse. La structure très variable des syllabes leur donne plus ou moins de volume. Dans d'autres contextes rythmiques, les mots pourraient être scandés différemment.

\section{Syllabes toniques portant naturellement le TF}

1. Monosyllabe tonique suivi d'un e atone non élidé :

"Chante, (déesse, l'ire)..." (Il. 1.1)

2. Monosyllabe tonique :

"Qui (des dieux)... ?»(Il. 1.8)

3. Syllabe à la fin d'un mot de 2, 3, ou 4 syllabes, ou davantage :

"... aux armées danaennes » (Il. 13.779)

« ... achevant l'idée... » $[I l .1,5(1,5)]$

"(A)gamemnon, fils d'Atrée... » (Il. 9.315)

4. La syllabe finale de groupe de mots (notion de mot étendue) devant ponctuation est nécessairement tonique ; l'avant-dernière syllabe, si la finale contient un e atone :

"Fils de Tydée, Diomède !...." (Il. 6.235)

"Ah ! le terrible vieillard! toujours, sans arrêt, tu travailles!" (Il. 10.164)

On trouve une exception à cette règle, dans notre Iliade:

" tard-venu, tard accompli » etc. (Il. 2.325)

Ce genre de recul de l'accent, rare ou exceptionnel, permet d'avoir des motsdactyles en français. En réalité, il n'y a de mot-dactyle en français que dans un ensemble de quatre syllabes, dont la finale est marquée : tard-venu-tard. Ici, l'effacement de l'accent final par intégration dans un ensemble élargi éloigne de la prosodie de la langue parlée, qui reste celle qu'on applique à l'hexamètre, et orienterait la diction dans un sens plus musical du phrasé.

De même, une fin de groupe de mots est tonique, en principe, même sans ponctuation :

"Puis Mérion offrit un-carquois et-un-arc à-Ulysse » (Il. 10.260) 
5. La syllabe initiale d'un mot de trois syllabes peut recevoir un accent complémentaire. L'expression de cette syllabe secondairement accentuée est délicate. Si on neutralise cet accent, on perd le relief souhaité. Si on exagère l'accent ou la durée, on risque de quitter le registre de la langue parlée :

"ouvragé dans le cuir..." (Il. 10.262)

"Puisque aujourd'hui les destinées de la mort nous échappent... " (Il. 12.326)

"Il fit sa proie de Thersiloque...." (Il. 21.209)

"Face à eux, les Troyens, sur le mamelon de la plaine... " (Il. 20.3)

«attends-le dans la multitude, au sortir du tumulte» (Il. 20.377).

On notera la différence d'accroche, pour l'accent complémentaire, entre une syllabe ouverte marquée (mamelon : $\mathrm{CV \# )} \mathrm{et} \mathrm{une} \mathrm{syllabe} \mathrm{fermée} \mathrm{ou} \mathrm{une} \mathrm{syllabe} \mathrm{à}$ liquide devant consonne (destinées, Thersiloque, multitude : CVL\#).

6. La syllabe initiale d'un mot de quatre syllabes doit recevoir un accent complémentaire pour que le mot s'intègre à l'hexamètre, où l'on ne peut avoir plus de deux syllabes non-marquées :

"Agamemnon s'en fut... " (Il.11.264)

" Il vous arrache la vie de son extrémité pénétrante! " (Il. 11.392)

«Puis Aisymnos, Oros et Hipponoos cœur-farouche! » (Il. 11.303)

Noter encore sur " extrémité » l'accroche de la syllabe complexe (EXT\#), plus apte à recevoir un accent complémentaire.

Même accent complémentaire à l'initiale d'un groupe de quatre syllabes :

"Soyez des braves! Rappelez-vous votre fougue vaillante! " (Il. 11.287)

7. Parfois, la $2^{\mathrm{e}}$ syllabe d'un mot long (on peut hésiter dans certains cas entre la $1^{\text {re }}$ et la $2^{\mathrm{e}}$ d'un mot long) reçoit l'accent complémentaire; ce fait est souvent lié à la présence d'un TF juste avant le mot long :

"les rangées de Troyens, progressivement, s'avançèrent. » (Il. 11.412)

" ni dans le campement d'Agamemnon l'Atréide " (Il. 9.226)

Parfois, on hésite à accentuer la première ou la seconde syllabe d'un mot long: "à écarter des embarcations les flammes voraces " (Il. 9.347)

Le choix se fait peut-être sur la qualité de la syllabe complexe \#BAR\#C, plus apte à accrocher la marque, moins facile à réduire en "brève ".

8. On rencontre un mot de six syllabes triplement accentué, avec jeu sonore [gn] : "Ignominieusement l'entraînaient aux nefs achéennes... » (Il. 22.465)

Là, il semble que des raisons expressives entraînent des phénomènes de dilatation au service de la quantité syllabique. 
Le jeu d'opposition marqué / non marqué renforce des oppositions sonores en plus d'une occasion, avec un effet d'onomatopée : «On ne gagne rien à la lamentation lamentable " (Il. 24.524).

\section{Syllabe devant porter un TF par sa place}

1. En première position dans l'hexamètre, toute syllabe, quelle qu'elle soit (l'accent rythmique imposant le TF à la discrétion de l'aède même si la syllabe n'est pas tonique), est marquée, que le mot ait une, deux syllabes ou davantage :

« le Péléide aplanit les braises... » (Il. 9.213)

"Je te prends les genoux..." (Il. 21.74)

"Priam a pris son épouse...» (Il. 21.88)

"Polydore divin..." (Il. 21.91)

Sur l'article "le ", il faut noter que l'accentuation de la syllabe initiale de l'énoncé métrique induit un renforcement tout à fait rare dans la prosodie naturelle, sauf effet d'insistance, didactique ou autre. Son épicisation, son accession à la dignité du commencement épique, la semnotês dont parle le musicographe antique Aristide Quintilien, sont-elles possibles? Je crois que c'est là un point essentiel qui tend à fonder ici le potentiel quantitatif, plus qu'intensif, de la marque rythmique. Le point le plus faible de la langue, la syllabe la plus atone, peut se voir investie du TF.

On note le déplacement d'accent sur les dissyllabes : d'oxyton, le mot devient paroxyton. Jeu ici renforcé ou facilité par l'allitération ("Priam a pris...»), avec un jeu sur l'intonation propre au chant de la langue orale, son accentus.

On note que le trisyllabe "Polydor(e) » peut être accentué ailleurs comme un anapeste et l'est ici comme un crétique, compatible avec un début d'hexamètre. Cette valeur de crétique le rendrait aussi bien compatible avec un début de vers trochaïque. Pour obtenir un hexamètre parfaitement régulé, il faudrait tenir la syllabe centrale de «Polydor(e)».

2. Toujours tonique est l'avant-dernière syllabe du vers (avant l'e atone final, qui est le seul moyen de réaliser, dans la langue parlée, la syllabe atone finale de l'hexamètre) ; toutes les fins de vers auront donc cette structure de l'avant-syllabe finale ; on parlera aussi bien de syllabe finale féminine :

"...près des sveltes navires " (Il. 20.1)

À cette syllabe s'arrête, dans la versification de la langue française (celle en tout cas que je parle ${ }^{10}$ ), le compte des syllabes. La muette finale, dont l'existence si particulière

10 Dans un usage lyrique de la langue (celui des poètes de la Renaissance, ou d'un Francis Cabrel aujourd'hui), la marque est déportée en amont d'une syllabe, à la manière de 
reste un des enjeux majeurs de toute poésie en français, n'étant pas comptée, l'hexamètre français comprend donc entre onze et seize syllabes.

Par exception, un monosyllabe non-tonique pourrait-il intervenir à la place de la muette finale ? Je l'ai tenté une ou deux fois. Mais ça devient un enjambement entre côla plutôt qu'une véritable fin de vers.

3. En première position de pied (position relative), il faut nécessairement une syllabe marquée; c'est la visée de l'hexamètre que d'établir ces syllabes marquées entre lesquelles une ou deux syllabes non-marquées s'interposent. Il est à noter que dans la structure phonologique du français, ce point saillant est plus souvent amené par les syllabes qui le précèdent que posé au commencement d'un mot-dactyle, comme on l'a vu. Autrement dit, la structure iambique-anapestique de la langue paraît dominer dans l'hexamètre français. Le plus souvent, le TF vient après un temps faible (Tf) d'une ou deux syllabes. Toutefois, c'est aussi le cas dans l'hexamètre grec après la césure au TF (coupe au demi-pied) ou après la césure trochaïque.

4. À la césure (celle-ci se confondant avec la fin d'un groupe de mots), la syllabe finale est nécessairement marquée : en fait, la syllabe finale accentuée marque l'hexamètre de points naturels de césure. Quand on dit que le décasyllabe est césuré à 4 , on signifie d'une autre manière que la première marque intervient de manière constante à la quatrième syllabe. L'hexamètre développe cette affinité de l'accent et de la césure : une césure flottante d'un point de vue syllabique, puisque les segments sont de longueurs différentes; mais une césure constante du point de vue du mètre. La diérèse (coupe après un pied complet) après un dactyle, c'est-à-dire après deux syllabes non-marquées, n'existe qu'en synaphie, puisque tout groupe se termine par une syllabe marquée. Autrement dit, on a des césures au TF, quand la fin de mot est masculine, au Tf quand elle est féminine ${ }^{11}$. L'hexamètre avance par vagues.

\section{Syllabe susceptible de porter un Tf par sa nature}

1. La syllabe dont le noyau est constitué d'un e atone (non élidé) en fin de mot (je ne fais pas d'exception à cette règle dans l'hexamètre) est toujours non-marquée ; dans la poésie lyrique-dramatique, on peut s'amuser à relever dans certaines conditions ce genre de syllabe.

certains parlers régionaux : « au fond du jar-din... ». On utilisera cette possibilité pour les ioniques mineurs du théâtre, par exemple.

11 Parfois on trouve une diérèse entre deux pieds après un e muet ; il n'est pas sûr que cette coupe soit très heureuse. 
2. Les proclitiques, en général, ne sont pas marqués : le, la, les, $d e, d u$, des, les prépositions, viennent s'unir au mot qu'ils précèdent et ont par conséquent vocation à prendre place au Tf. Mais ces proclitiques peuvent parfois servir de relais à un "TF ", car un proclitique peut très bien porter le TF imposé par l'alternance rythmique, comme on le voit en début de vers ou à une autre place contraignante du vers. Étonnante plasticité de la langue française : la faiblesse qu'on lui reproche fait aussi sa force. Comme en grec, une particule pourra être tour à tour marquée et non-marquée dans le même vers.

3. Corollaire de la proposition précédente, un monosyllabe intégré à un groupe phonologique (hormis en place finale) et par conséquent placé en amont de la syllabe marquée est nécessairement non-marqué.

4. Est non-marquée la (ou les deux) syllabe(s) précédant, dans un mot, la syllabe finale marquée.

5. Dans les principes qui régissent l'établissement de l'hexamètre français, une syllabe faible par son statut peut toutefois être riche par sa structure. Ainsi, dans l'expression de fin d'hexamètre "égal à l'obscure bourrasque ", les syllabes sises au Tf, en admettant cette division syllabique approximative, l'OBS\# ou BOUR\#, pourraient être, ailleurs, dans un autre contexte prosodico-métrique, marquées au TF, mais doivent ici être maintenues en " brève " au Tf.

De même, dans ce début d'hexamètre "Seigneur des morts, Aidoneus... ", le marquage de la syllabe initiale est artificiel, mais doit en plus s'accompagner d'une minoration de la finale, riche (GNEUR\#/D), potentiellement « lourde ${ }^{12}$ » : régulation assez difficile, peut-être due au potentiel plus naturellement iambique du mot. On en dirait autant de la diphtongue initiale du nom Aidoneus, en position de "brève " au Tf. La régulation aédique qui est censée s'opérer consiste en un abrègement, ou tout au moins en un maintien de l'opposition structurante entre demi-pied faible (une ou deux syllabes) et demi-pied fort (une syllabe unique). Il y a là tout un champ de possibles qui s'ouvre à la composition, dans une écoute plus ou moins attentive à la nature de la langue, ainsi qu’à la récitation, plus ou moins contrainte par la mesure.

12 Si l'on recourt au couple terminologique heavy / light de W.S. Allen, Accent and Rythm, Cambridge 1973. 


\section{Syllabe obligatoirement faible par sa place}

Du point de vue du mètre, et non plus seulement du point de vue de la langue, toute syllabe placée juste après ou juste avant un temps fort est faible.

Après la première syllabe d'un groupe de deux au temps faible, toute syllabe est faible.

\section{En guise de conclusion}

Avec les règles « de nature » on arrive à plusieurs possibilités de marque accentuelle. La première version du programme de scansion Scande đoMarque écrit par Gilles de Rosny pour scander l'hexamètre français rencontre parfois des difficultés pour déterminer la marque dans les suites de monosyllabes.

Ces règles doivent être complétées par les règles « de place " (ou de position) dans le mot ou le groupe de mots. Avec les règles de "place » dans la structure de l'hexamètre, on réduit ces possibilités : après un TF, il y a toujours une ou deux syllabes au Tf précédant un autre TF, etc.

La principale règle de régulation impose de ne pas marquer une syllabe qui ne doit pas l'être. Ainsi, au commencement du vers, on évitera de marquer la deuxième syllabe. On peut à la rigueur neutraliser la marque sur la syllabe initiale de l'hexamètre, mais il importe d'avoir la deuxième marque sur la troisième ou la quatrième syllabe.

$\mathrm{Si}$ on devait admettre de laisser une liberté de quantité aux deux premières syllabes du vers, on obtiendrait pour l'hexamètre français le genre de liberté qui existe dans la métrique éolienne d'Alcée et Sappho. Ce qui, à tout prendre, serait également intéressant.

En tout cas, l'expérience d'hexamètre iambo-anapestique avec anacrouse, développée par Jean Tardieu pour traduire Hölderlin ${ }^{13}$, n'a pas été suivie dans les traductions de Catulle, d'Hésiode ou de l'Iliade récemment publiées. Le problème de la marque initiale a rencontré deux solutions dès la traduction de Catulle par André Markowicz : il convenait ou bien de valoriser l'accent initial quand celui-ci existe, ou bien de mettre ce premier accent défaillant entre parenthèses. On avait ainsi une première marque facultative. Ma position aujourd'hui est qu'on peut valoriser la marque initiale quel que soit le mot, en orientant l'hexamètre français vers une scansion plus quantitative ou plus intensive, selon la qualité de la syllabe.

Du reste, la marque absente l'est-elle vraiment ? Dans la diction d'un poème épique, l'art consiste à effacer ou à souligner la mécanique, à valoriser la langue ou le mètre dans une contradiction productrice de mémoire. Au début, la langue peut paraître s'opposer au mètre. La langue devient d'abord matière à discipliner par le mètre, puis 
l'objet du poème devient cette matière scandée elle-même, élément métrique, côlon allitérant, ou matrice sonore. L'effet de récurrence rythmique permet ici d'effacer un contour, là de suppléer la marque. La marque, d'une manière ou d'une autre, même par défaut, est toujours là. N'oublions pas qu'il s'agit d'un mélos musical pris dans un flux continu et que la marque rythmique, dans la parole comme dans le geste de la danse, se satisfait parfois d'être seulement mémoire de la marque à cette place.

Il est à noter que, pour traduire les hexamètres de Hölderlin ou de Goethe, euxmêmes imités de l'Antique, le travail de mise en rythme est exactement le même que pour traduire Homère. Voici le texte des premiers vers de l'Archipel, dans ma traduction en cours :

Migreront-elles vers toi, les grues, et vers tes rivages les bateaux voudront-ils recroiser, et les brises fidèles, caresser tes flots apaisés, et du fond de l'abîme, le dauphin venir tendre son dos à la neuve lumière ?

Est-il temps, pour l'Ionie, de fleurir ? Que vers toi je revienne au printemps, quand le cœur des amants est neuf, quand s'éveillent les premiers émois, quand les âges d'or se souviennent, à tout jamais! Salut, l'Ancien, en douceur et silence ! Tout-puissant, toujours tu vis tranquille dans l'ombre de tes montagnes, comme autrefois : de tes bras de jeune homme tu embrasses, Père, ton continent et tes filles, puisque aucune des îles, des florissantes, ne manque...

Aucune des îles ne manque. Complétude du vers prolongé, transféré, mimétiquement réinstauré, réactualisé dans sa scansion. L'Archipel redéploie ses bras dans l'hexamètre, recommencé plutôt que retrouvé. Nulle origine dont il faudrait exalter le principe. Nulle nuit indo-européenne qui en hébergerait l'être ! Il faut lire en Hölderlin le poète universel du Rousseau, plutôt que de l'abîmer dans l'obscurantisme des ontologues nazis. Homère, maitre en hospitalité, accueille Goethe, Schiller, Hölderlin ; accueille Mousset, Voss, Gneditch et les autres traducteurs à venir. De même, sans qu'il faille être analyste, Homère a toujours été là, précédé par lui-même, dans la battue de l'hexamètre. Pas plus qu'Homère, poète de la voix en tant qu'épos, n'existe dans les scansions muettes, l'hexamètre ne saurait se déployer ailleurs que dans le souffle de celui qui les dit, en prolonge les syllabes, en reprend et modifie les syntagmes, en prépare activement la migration vers d'autres horizons linguistiques. Homère, fils du fleuve, ne vaut que par son devenir.

Philippe BRUNET

Professeur à l'Université de Rouen

Faculté des lettres et sciences humaines

76821 Mont-Saint-Aignan cedex

demodocos@demodocos.com 


\section{Dossier bibliographique sur l'épopée en traduction}

\section{Traductions métriques de l'épopée}

A. Markowicz (trad.), Le Livre de Catulle, L'Âge d'Homme, Paris 1985 [traduction intégrale des poèmes de Catulle selon les différents mètres, avec une Introduction et une Note du traducteur ; les poèmes 63 (Attis), 64 (Noces de Thétis et Pélée), 68 (Protésilas), entre autres, sont souvent dits par la compagnie Démodocos].

Ph. Brunet (trad.), La Batrachomyomachie d'Homère, texte établi par Y. MigouberT, Allia, Paris 1998 [l'épopée parodique attribuée à Homère].

Ph. Brunet (trad.), Hésiode, La Théogonie. Les Travaux et les jours et autres poèmes, Le Livre de Poche, Paris 1999 [les hexamètres du grand rival d'Homère]

Ph. Brunet (trad.), Homère, L'Iliade, Seuil, Paris 2010, Point Seuil, Paris 2012 [spectacle L'Iliade 2000 ; lectures intégrales à Paris, Avignon, Athènes 2005-06 et récitals divers ; récemment: Arches troyennes en Arques, Arques-la-Bataille 2014].

En cours de traduction et donnés en lecture ou en spectacle

Ph. Brunet (trad.), Homère, L'Odyssée [spectacles de la compagnie Démodocos, Le Retour d'Ulysse 1995, Les Amours d'Arès et d'Aphrodite 1996, Circé 1999-2012, Preneur de Troie 2000, Cyclope 2007, Les errances d'Ulysse 2008 et lectures diverses].

A. MüNCH (trad.), Virgile, Géorgiques, extraits donnés dans le spectacle Orphée en 2000, lectures en 2011 et 2012.

G. Boussard (trad.), Lucrèce, La Nature des choses, extraits donnés en récital piano-voix par G. Boussard et E. Lascoux (2010-13).

Prosodie, mètre, rythme, danse (classement plus ou moins chronologique)

P. SeRVIEn, Essai sur les rythmes toniques du Français, Paris 1925.

M. C. GHYkA, Le nombre d'or, chapitre " Du rythme à l'incantation », Paris 1931, p. 99-144.

A. Dain, Traité de métrique grecque, Paris 1965.

E. ETKIND, Un art en crise. Essai de critique de la traduction poétique, L'Âge d'Homme 1982.

J. Irigoin, Le poète grec au travail, Mémoires de l'Académie 39, Paris 2009.

Ph. Brunet, La naissance de la littérature dans la Grèce ancienne, LGF, Paris 1997.

Ph. Brunet, "Le grec, langue idéale du chant ", Musique et poésie dans l'Antiquité. Actes du colloque de Clermont-Ferrand (23 mai 1997), éd. G. Pinault, Université Blaise Pascal, Clermont-Ferrand 2001, p. 11-26. 
Ph. Brunet, «Écrit sur la voix », Les chants d'Orphée. Musique et poésie (dir. C. PeILlon), La Pensée de midi, 28, 2009, p. 22-29.

$\mathrm{Ph}$. BRUNET, "Mètre et danse : pour une interprétation chorégraphique des mètres grecs ", Poesia, musica e agoni nella Grecia antica, Atti del IV convegno di MOISA, Lecce 28-30 ottobre 2010, Rudiae 22-23, t. II, publié en 2012, p. 557-571 [sur la dimension orchestique de la poésie grecque].

Ph. BRunEt, «La voix et le geste au pouls de la Muse ", Approches n 150, juin 2012, p. $141-54$.

M. STEINRÜCK, À quoi sert la métrique? Interprétation littéraire et analyse des formes métriques grecques : une introduction, Grenoble 2006

G. DE Rosny, ScandeðChante, programme de scansion de l'hexamètre homérique en ligne sur www.homeros.fr, 2010.

G. De Rosny, ScandeむMarque, programme de scansion de l'hexamètre français en préparation pour le site www.homeros.fr, 2014.

Autour de la publication écrite et orale de l'Iliade en hexamètres :

M. Nappi et J.-Fr. Puff, "Le temps suspendu de l'Iliade », entretien avec Ph. B., p. 75-85, Geste, 4, 2007.

G. BeLZANE, «L'épopée, c'est la voix », entretien avec Ph. B., TDC999, 2010, p. 20-21.

$\mathrm{Ph}$. BRUnET, "Traduire en hexamètres, redire Homère ", La retraduction (dir. R. KAHN, C. Seth), Rouen-Le Havre, 2010, p. 243-51.

Ch. FicAT, "Les colonnes d'Homère ", p. 72-77 ; "L'Iliade, une nouvelle parole épique ", entretien avec Ph. B., p. 78-85, Revue des deux mondes, fév. 2011.

$\mathrm{Ph}$. BRUnet, "Sur la traduction rythmée d'Homère ", résumé d'atelier, dans VingtHuitièmes Assises de la traduction littéraire, Arles 2011.

Ph. BRunet (éd.), "L'Iliade d'Homère par la compagnie Démodocos ", par B. BÉneCH ("La voix sacrée d'Homère »), Ph. B. ("Redire l' Iliade d'Homère »), E. Lascoux ("Reste le vers »), G. Boussard (" L'Iliade, format paysage »), P. Thébaud ("Composer pour l'Iliade d'Homère »), F. CAM ("Fragments épiques »), Ph. B. ( La composition de l'Iliade en 24 chants »), P. ForTASSIER ( Rigoureux parallélisme dans la composition de l'Iliade et de l'Odyssée »), dans le "Carrefour de la recherche ", Cahier de Poétique 11, 2006, Université Paris 8, p. 72-100 [sur la publication orale de l'Iliade en 2005 à la Sorbonne et à Avignon].

C. Le Meur, "Une poétique monumentale. Les Psaumes et l'Iliade, traductions récentes ", Acta Fabula, Fabula.org, mis en ligne le 19 février 2012.

C. Noacco et A. Grand-Clément, "Homère, L'Iliade, traduit du grec par Philippe Brunet », Anabases, 16, 2012, p. 311-314, mis en ligne le $1^{\mathrm{er}}$ octobre 2012, http:// anabases.revues.org/4023.

M. Volkovitch, «Un saint chez Homère », Pages d'écriture 116, en mai 2013, http:// www.volkovitch.com/rub_carnet.asp?a=pe116. 